\title{
Thickness shear vibrations of a circular cylindrical piezoelectric shell
}

\author{
J. S. Yang \\ Department of Mechanical Engineering, Rensselaer Polytechnic Institute, Troy, New York 12180
}

R. C. Batra

Department of Engineering Science and Mechanics, Virginia Polytechnic Institute and State University, Blacksburg, Virginia 24061-0219

(Received 24 February 1994; revised 1 July 1994; accepted 22 August 1994)

\begin{abstract}
Axial and tangential thickness shear vibrations of a circular cylindrical piezoelectric shell of monoclinic crystals are studied. The problems are solved analytically, and the frequency equations are derived. For a cylinder made of a rotated Y-cut quartz, the resonant frequencies are computed numerically, and it is shown that they approach that of a flat plate as the inner radius of the cylinder of finite thickness approaches infinity.
\end{abstract}

PACS numbers: 43.40.Ey

\section{INTRODUCTION}

Thickness shear vibrations of crystals and piezoelectric plates have been studied either by using the threedimensional equations of piezoelectricity or the twodimensional equations of the plate theory. ${ }^{1-3}$ The interest in these problems arises because of their applications as resonators. Vibrations of a circular cylindrical piezoelectric shell, with deformations assumed to be either axisymmetric or with the tangential displacement taken to be zero, and made of ceramics poled in various directions, have also been studied. ${ }^{4-7}$ In this paper, axial and tangential thickness shear vibrations of a circular cylindrical piezoelectric shell made of a monoclinic crystal are studied. We derive exact solutions of the three-dimensional quasistatic piezoelectricity equations governing the free vibrations of a cylindrical shell with traction-free and electroded inner and outer surfaces. Frequency equations are also derived and solved numerically.

\section{THICKNESS SHEAR VIBRATIONS OF A PLATE}

Results for the thickness shear vibrations of a monoclinic piezoelectric plate ${ }^{2}$ are summarized below for easy reference. Consider an infinite plate, shown in Fig. 1, of thickness $2 h$ with traction-free and electroded boundaries at $x_{2}= \pm h$. Equations governing the deformations of the plate and the relevant boundary conditions are

$$
\begin{aligned}
& T_{j i, j}=\rho \ddot{u}_{i}, \quad-h<x_{2}<h, \\
& D_{i, i}=0, \quad-h<x_{2}<h, \\
& T_{2 i}=0, \quad \phi=0 \quad \text { at } x_{2}= \pm h,
\end{aligned}
$$

where

$$
\begin{aligned}
& T_{i j}=C_{i j k l} S_{k l}-e_{k i j} E_{k}, \\
& D_{i}=e_{i j k} S_{j k}+\epsilon_{i j} E_{j}, \\
& S_{i j}=\frac{1}{2}\left(u_{i, j}+u_{j, i}\right), \\
& E_{i}=-\phi_{, i} .
\end{aligned}
$$

In Eqs. (1)-(7) and hereafter, $T_{i j}, u_{i}, D_{i}, S_{i j}$, and $E_{i}$ are the components of the stress tensor, mechanical displacement, electric displacement, the strain tensor appropriate for infinitesimal deformations, and the electric field, respectively, $\rho$ and $\phi$ the mass density and the electric potential, and $C_{i j k l}$, $e_{i j k}$, and $\epsilon_{i j}$ the elastic, piezoelectric, and dielectric constants, respectively. Furthermore, a comma followed by index $j$ indicates partial differentiation with respect to $x_{j}$, a superimposed dot indicates differentiation with respect to time $t$, and a repeated index implies summation over the range of the index.

Equation (1) expresses the balance of linear momentum and Eq. (2) is the Gauss equation. The boundary conditions (3) ${ }_{1}$ and (3) $)_{2}$ imply that the bounding surfaces $x_{2}= \pm h$ are traction-free and have null electric potential prescribed there. Equations (4) and (5) are the constitutive relations and Eq. (6) is the strain-displacement relation; Eq. (7) is the electricfield-potential relation. Regarding $T_{i j}=T_{j i}$ and $S_{i i}=S_{j i}$ as vectors in a six-dimensional space with $T_{1}=T_{11}, T_{2}=T_{22}$, $\bar{T}_{3}=T_{33}, \bar{T}_{4}=T_{23}, \bar{T}_{5}=T_{31}$, and $\bar{T}_{6}=T_{12}$ etc., the material constants $C_{i j k l}$ and $e_{k i j}$ may be written as $6 \times 6$ and $6 \times 3$ matrices. For monoclinic crystals,

$$
\epsilon_{i j}=\epsilon_{j i}, \quad \epsilon_{12}=\epsilon_{13}=0 .
$$

For the free-time-harmonic thickness shear vibrations in the $x_{1}$ direction, we seek solutions satisfying

$$
\begin{aligned}
& u_{1}=\tilde{u}_{1}\left(x_{2}\right) e^{i \omega t}, \quad u_{2}=0, \quad u_{3}=0, \\
& \phi=\tilde{\phi}\left(x_{2}\right) e^{i \omega t} .
\end{aligned}
$$

Equations (1)-(7) become

$$
\begin{aligned}
& T_{21,2}=-\rho \omega^{2} u_{1}, \quad D_{2,2}=0, \quad-h<x_{2}<h, \\
& T_{21}=0, \quad \phi=0 \quad \text { at } x_{2}= \pm h, \\
& T_{31}=2 C_{56} S_{12}-e_{25} E_{2}, \quad T_{12}=2 C_{66} S_{12}-e_{26} E_{2}, \\
& D_{2}=2 e_{26} S_{12}+\epsilon_{22} E_{2}, \quad D_{3}=2 e_{36} S_{12}+\epsilon_{23} E_{2}, \\
& S_{12}=\frac{1}{2} u_{1,2}, \quad E_{2}=-\phi_{, 2},
\end{aligned}
$$




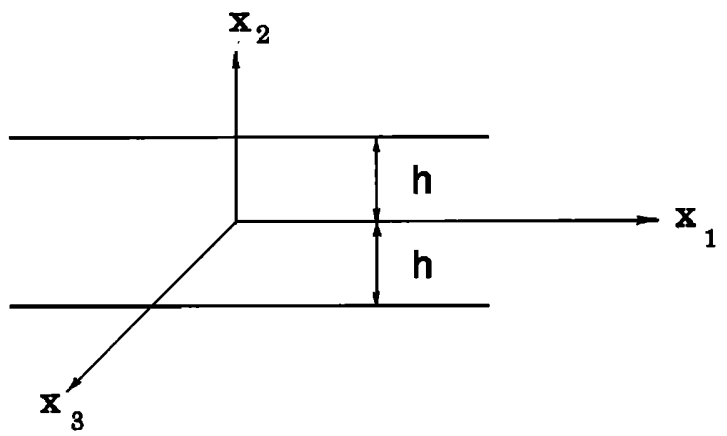

FIG. 1. Schematic sketch of a piezoelectric plate.

wherein we have dropped the tildes superimposed upon $u_{1}$ and $\phi$. The general solution of (10) is

$$
\begin{aligned}
& u_{1}=a_{1} \sin k x_{2}+a_{2} \cos k x_{2}, \\
& \phi=\frac{e_{26}}{\epsilon_{22}}\left(a_{1} \sin k x_{2}+a_{2} \cos k x_{2}\right)+a_{3} x_{2}+a_{4},
\end{aligned}
$$

where

$$
k^{2}=\rho \omega^{2} / \bar{C}_{66}, \quad \bar{C}_{66}=C_{66}+e_{26}^{2} / \epsilon_{22},
$$

and $a_{1}, a_{2}, a_{3}$, and $a_{4}$ are arbitrary constants. Substitution from (15) and (16) into boundary conditions (11) gives the following set of homogeneous equations for the determination of $a_{1}, a_{2}, a_{3}$, and $a_{4}$ :

$k\left(C_{66}\left(a_{1} \cos k h-a_{2} \sin k h\right)+\frac{e_{26}^{2}}{\epsilon_{22}}\left(a_{1} \cos k h\right.\right.$

$$
\left.\left.-a_{2} \sin k h\right)\right)+e_{26} a_{3}=0
$$

$k\left(C_{66}\left(a_{1} \cos k h+a_{2} \sin k h\right)+\frac{e_{26}^{2}}{\epsilon_{22}}\left(a_{1} \cos k h\right.\right.$

$$
\left.\left.+a_{2} \sin k h\right)\right)+e_{26} a_{3}=0
$$

$\frac{e_{26}}{\epsilon_{22}}\left(a_{1} \sin k h+a_{2} \cos k h\right)+a_{3} h+a_{4}=0$,

$\frac{e_{26}}{\epsilon_{22}}\left(-a_{1} \sin k h+a_{2} \cos k h\right)-a_{3} h+a_{4}=0$.

The vanishing of the determinant of the coefficient matrix of Eqs. (18)-(21) gives the following frequency equation:

$$
\sin k h\left(\tan k h-k h / k_{26}^{2}\right)=0, \quad k_{26}^{2}=e_{26}^{2} / \bar{C}_{66} \epsilon_{22} .
$$

\section{AXIAL THICKNESS SHEAR VIBRATIONS OF A CIRCULAR CYLINDRICAL SHELL}

We consider a cylindrical shell, shown in Fig. 2, made of a monoclinic crystal and with inner radius $a$ and outer radius $b$. It is more convenient to use cylindrical coordinates, and we refer the reader to Love's book ${ }^{8}$. for the governing equations (1) and (2); constitutive relations (4), and straindisplacement relations (6) written in cylindrical coordinates. It is preferable to work in terms of physical components of

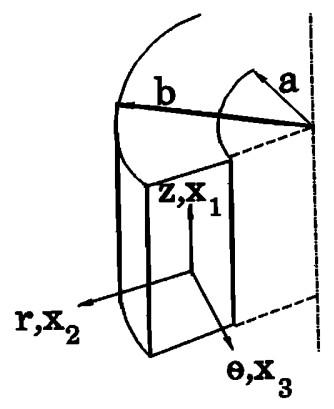

FIG. 2. A circular cylindrical shell and the choice of base vectors for studying its axial thickness shear vibrations.

stresses, strains, and the electric displacement. The constitutive relations (4) and (5), and electric-field-potential relations (7) become

$$
\begin{aligned}
& T_{z z}=C_{11} S_{z z}+C_{12} S_{r r}+C_{13} S_{\theta \theta}+2 C_{14} S_{r \theta}-e_{11} E_{z} \\
& T_{r r}=C_{12} S_{z z}+C_{22} S_{r r}+C_{23} S_{\theta \theta}+2 C_{24} S_{r \theta}-e_{12} E_{z}, \\
& T_{\theta \theta}=C_{13} S_{z z}+C_{23} S_{r r}+C_{33} S_{\theta \theta}+2 C_{34} S_{r \theta}-e_{13} E_{z} \\
& T_{r \theta}=C_{14} S_{z z}+C_{24} S_{r r}+C_{34} S_{\theta \theta}+2 C_{44} S_{r \theta}-e_{14} E_{z}, \\
& T_{\theta z}=2 C_{55} S_{\theta z}+2 C_{56} S_{z r}-e_{25} E_{r}-e_{35} E_{\theta}, \\
& T_{z r}=2 C_{56} S_{\theta z}+2 C_{66} S_{z r}-e_{26} E_{r}-e_{36} E_{\theta}, \\
& D_{z}=e_{11} S_{z z}+e_{12} S_{r r}+e_{13} S_{\theta \theta}+2 e_{14} S_{r \theta}+\epsilon_{11} E_{z} \\
& D_{r}=2 e_{25} S_{\theta z}+2 e_{26} S_{z r}+\epsilon_{22} E_{r}+\epsilon_{23} E_{\theta}, \\
& D_{\theta}=2 e_{35} S_{\theta z}+2 e_{36} S_{z r}+\epsilon_{23} E_{r}+\epsilon_{33} E_{\theta} \\
& E_{r}=-\frac{\partial \phi}{\partial r}, \quad E_{\theta}=-\frac{1}{r} \frac{\partial \phi}{\partial \theta}, \quad E_{z}=-\frac{\partial \phi}{\partial z}
\end{aligned}
$$

For the free-time-harmonic thickness shear vibrations in the axial direction, we seek solutions of the form

$$
u_{z}=\bar{u}_{z}(r) e^{i \omega t}, \quad u_{r}=0, \quad u_{\theta}=0, \quad \phi=\tilde{\phi}(r) e^{i \omega t} .
$$

With (24), and dropping the tildes superimposed upon $u_{z}$ and $\phi$, the governing equations and the boundary conditions simplify to

$$
\begin{aligned}
& \frac{\partial T_{r z}}{\partial r}+\frac{T_{r z}}{r}=-\rho \omega^{2} u_{z}, \quad \frac{1}{r} \frac{\partial}{\partial r}\left(r D_{r}\right)=0, \quad a<r<b \\
& S_{z r}=\frac{1}{2} \frac{\partial u_{z}}{\partial r}, \quad E_{r}=-\frac{\partial \phi}{\partial r} \\
& T_{r z}=2 C_{66} S_{r z}-e_{26} E_{r}, \quad D_{r}=2 e_{26} S_{r z}+\epsilon_{22} E_{r}, \\
& T_{\theta z}=2 C_{55} S_{\theta z}-e_{25} E_{r}, \quad D_{\theta}=2 e_{36} S_{r z}+\epsilon_{23} E_{r} \\
& T_{r z}=0, \quad \phi=0 \quad \text { at } r=a, b
\end{aligned}
$$

Substitution from (26) and (27) into (25) yields

$$
\begin{aligned}
& \frac{\partial}{\partial r}\left(C_{66} u_{z, r}+e_{26} \phi_{, r}\right)+\frac{1}{r}\left(C_{66} u_{z, r}+e_{26} \phi_{, r}\right) \\
& =-\rho \omega^{2} u_{z},
\end{aligned}
$$




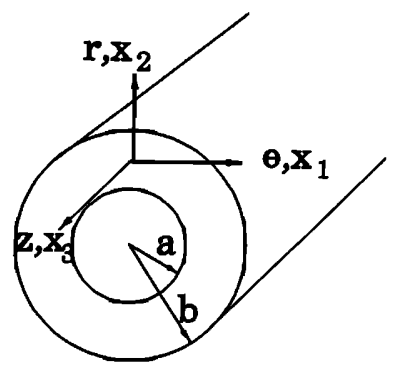

FIG. 3. A circular cylindrical shell and the choice of base vectors for studying its tangential thickness shear vibrations.

$\frac{\partial}{\partial r}\left(e_{26} u_{z, r}-\epsilon_{22} \phi_{, r}\right)+\frac{1}{r}\left(e_{26} u_{z, r}-\epsilon_{22} \phi_{, r}\right)=0$.

The elimination of $\phi$ from (29) and (30) gives

$$
u_{z, r r}+\frac{1}{r} u_{z, r}+k^{2} u_{z}=0
$$

where

$$
k^{2}=\rho \omega^{2} / \bar{C}_{66}, \quad \bar{C}_{66}=C_{66}+e_{26}^{2} / \epsilon_{22} .
$$

Solving Eqs. (31) and (30) for $u_{z}$ and $\phi$, we obtain

$$
\begin{aligned}
& u_{z}=C_{1} J_{0}(k r)+C_{2} Y_{0}(k r), \\
& \phi=\frac{e_{26}}{\epsilon_{22}} u_{z}+\frac{e_{26}}{\epsilon_{22}}\left(C_{3} \ln r+C_{4}\right),
\end{aligned}
$$

where $J_{0}$ and $Y_{0}$ are zeroth-order Bessel's functions of the first and second kind, respectively, and constants $C_{1}, C_{2}, C_{3}$, and $C_{4}$ are to be determined from the boundary conditions. The requirement that functions $u_{z}$ and $\phi$ satisfy boundary conditions (28) give four homogeneous equations for the determination of $C_{1}, C_{2}, C_{3}$, and $C_{4}$. These four equations have a nontrivial solution only if

$$
\begin{aligned}
& \frac{k a J_{1}(k a) \ln a / b+k_{26}^{2}\left[J_{0}(k a)-J_{0}(k b)\right]}{k b J_{1}(k b) \ln a / b+k_{26}^{2}\left[J_{0}(k a)-J_{0}(k b)\right]} \\
& \quad=\frac{k a Y_{1}(k a) \ln a / b+k_{26}^{2}\left[Y_{0}(k a)-Y_{0}(k b)\right]}{k b Y_{1}(k b) \ln a / b+k_{26}^{2}\left[Y_{0}(k a)-Y_{0}(k b)\right]},
\end{aligned}
$$

where relations $J_{0}^{\prime}=-J_{1}, Y_{0}^{\prime}=-Y_{1}$ have been used; $J_{1}$ and $Y_{1}$ are first-order Bessel's functions of the first and second kind, respectively. Equation (35) is the equation for the determination of the frequency $k$, and thence $\omega$ through $\mathrm{Eq}$. $(32)_{1}$.

\section{TANGENTIAL THICKNESS SHEAR VIBRATIONS OF A CIRCULAR CYLINDRICAL SHELL}

We now study the tangential thickness shear vibrations of a cylindrical shell made of a monoclinic crystal aligned as shown in Fig. 3 wherein the coordinate system is also depicted. With respect to the coordinate axes shown, the constitutive relations take the form

$$
\begin{aligned}
& T_{\theta \theta}=C_{11} S_{\theta \theta}+C_{12} S_{r r}+C_{13} S_{z z}+2 C_{14} S_{r z}-e_{11} E_{\theta}, \\
& T_{r r}=C_{12} S_{\theta \theta}+C_{22} S_{r r}+C_{23} S_{z z}+2 C_{24} S_{r z}-e_{12} E_{\theta},
\end{aligned}
$$

$$
\begin{aligned}
& T_{z z}=C_{13} S_{\theta \theta}+C_{23} S_{r r}+C_{33} S_{z z}+2 C_{34} S_{r z}-e_{13} E_{\theta}, \\
& T_{r z}=C_{14} S_{\theta \theta}+C_{24} S_{r r}+C_{34} S_{z z}+2 C_{44} S_{r z}-e_{14} E_{\theta}, \\
& T_{z \theta}=2 C_{35} S_{z \theta}+2 C_{56} S_{\theta r}-e_{25} E_{r}-e_{35} E_{z}, \\
& T_{\theta r}=2 C_{56} S_{z \theta}+2 C_{66} S_{\theta r}-e_{26} E_{r}-e_{36} E_{z}, \\
& D_{\theta}=e_{11} S_{\theta \theta}+e_{12} S_{r r}+e_{13} S_{z z}+2 e_{14} S_{r z}+\epsilon_{11} E_{\theta}, \\
& D_{r}=2 e_{25} S_{\theta z}+2 e_{26} S_{r \theta}+\epsilon_{22} E_{r}+\epsilon_{23} E_{z}, \\
& D_{z}=2 e_{35} S_{\theta z}+2 e_{36} S_{r \theta}+\epsilon_{23} E_{r}+\epsilon_{33} E_{z} .
\end{aligned}
$$

We assume that the free-time-harmonic thickness shear vibrations in the tangential direction are given by

$$
u_{\theta}=\tilde{u}_{\theta}(r) e^{i \omega t}, \quad u_{r}=0, \quad u_{z}=0, \quad \phi=\tilde{\phi}(r) e^{i \omega t} .
$$

For motions of this type, the governing equations and boundary conditions simplify to

$$
\begin{aligned}
& \frac{\partial T_{r \theta}}{\partial r}+\frac{2}{r} T_{r \theta}=-\rho \omega^{2} u_{\theta}, \quad \frac{1}{r} \frac{\partial}{\partial r}\left(r D_{r}\right)=0, \\
& S_{r \theta}=\frac{1}{2}\left(u_{\theta, r}-\frac{u_{\theta}}{r}\right), \quad E_{r}=-\phi_{, r}, \\
& T_{z \theta}=2 C_{56} S_{r \theta}-e_{25} E_{r}, \quad T_{\theta r}=2 C_{66} S_{r \theta}-e_{26} E_{r}, \\
& D_{r}=2 e_{26} S_{r \theta}+\epsilon_{22} E_{r}, \quad D_{z}=2 e_{36} S_{r \theta}+\epsilon_{33} E_{r}, \\
& T_{r \theta}=0, \quad \phi=0 \quad \text { at } r=a, b,
\end{aligned}
$$

where we have dropped the tildes superimposed upon $u_{\theta}$ and $\phi$. A solution of Eq. $(38)_{2}$ is

$$
D_{r}=e_{26} C_{3} / r
$$

where $C_{3}$ is an arbitrary constant. Equations (43), $(41)_{1}$, and $(39)_{2}$ result in

$$
\phi, r=\frac{e_{26}}{\epsilon_{22}}\left(2 S_{r \theta}-\frac{C_{3}}{r}\right)
$$

Substitution from (40), (39) $)_{1}$, and (44) into (38) $)_{1}$ yields

$$
u_{\theta, r r}+\frac{1}{r} u_{\theta, r}+\left(k^{2}-\frac{1}{r^{2}}\right) u_{\theta}=\frac{k_{26}^{2} C_{3}}{r^{2}}
$$

where $k$ and $k_{26}$ are defined by Eqs. (32) 1 and (22) 2 , respectively. A solution of Eq. (45) is

$$
u_{\theta}=C_{1} J_{1}(k r)+C_{2} Y_{1}(k r)+C_{3} P(k r),
$$

where $C_{1}$ and $C_{2}$ are arbitrary constants, and

$$
\begin{aligned}
& P(k r)=\frac{\pi}{2} k_{26}^{2}\left[Y_{1}(k r) F(k r)-J_{1}(k r) G(k r)\right] \\
& F(k r)=\int_{k a}^{k r} \frac{J_{1}(\xi)}{\xi} d \xi,-G(k r)=\int_{k a}^{k r} \frac{Y_{1}(\xi)}{\xi} d \xi .
\end{aligned}
$$

Substituting from (39), and (46) into (44), and integrating the resulting equation, we obtain

$$
\begin{aligned}
\phi= & \frac{e_{26}}{\epsilon_{22}}\left\{C_{1}\left[J_{1}(k r)-F(k r)\right]+C_{2}\left[Y_{1}(k r)-G(k r)\right]\right. \\
& \left.+C_{3}[P(k r)-Q(k r)-\ln r]+C_{4}\right\}
\end{aligned}
$$




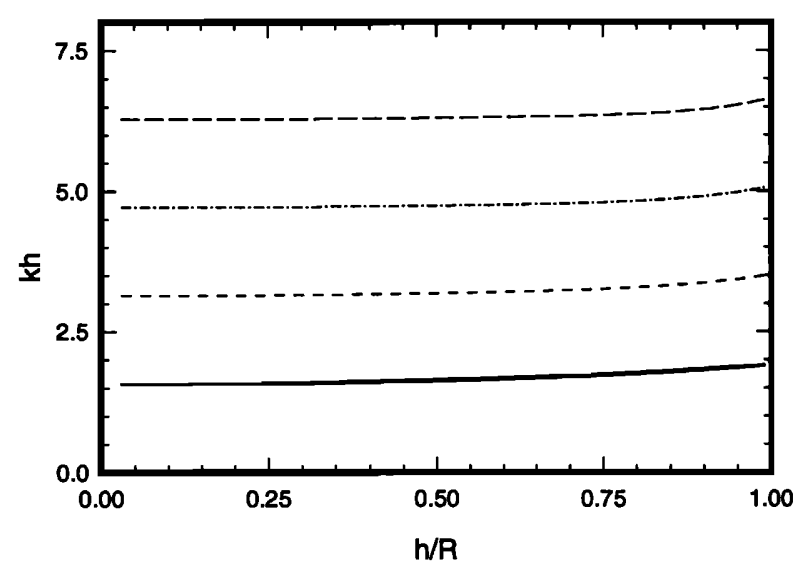

FIG. 4. First four resonant frequencies for axial thickness shear vibrations of

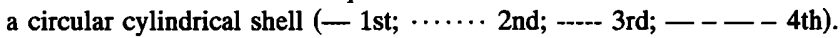

where

$$
Q(k r)=\int_{k a}^{k r} \frac{P(\xi)}{\xi} d \xi
$$

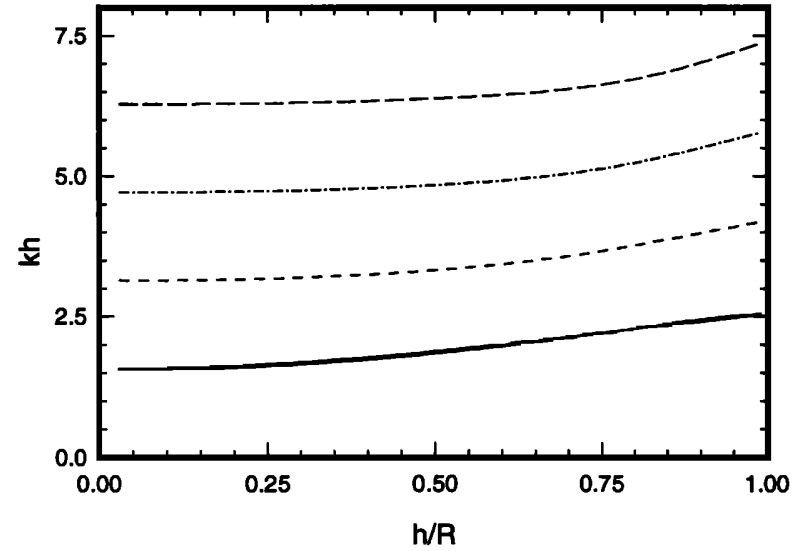

FIG. 5. First four resonant frequencies for tangential thickness shear vibrations of a circular cylindrical shell ( $(-1 \mathrm{st} ; \cdots \cdots) 2 \mathrm{nd}$; -.-- $3 \mathrm{rd}$; - - - 4th).

The four homogeneous algebraic equations for $C_{1}, C_{2}, C_{3}$, and $C_{4}$ obtained by substituting from (46) and (49) into the boundary conditions (42) will have a nontrivial solution only if

$$
\begin{gathered}
\frac{[P(k b)-Q(k b)+\ln a / b] k a J_{2}(k a)+\left[J_{1}(k a)-J_{1}(k b)+F(k b)\right] k_{26}^{2}}{[P(k b)-Q(k b)+\ln a / b] k b J_{2}(k b)+\left[J_{1}(k a)-J_{1}(k b)+F(k b)\right]\left[P(k b)-k b P^{\prime}(k b)+k_{26}^{2}\right]} \\
=\frac{[P(k b)-Q(k b)+\ln a / b] k a Y_{2}(k a)+\left[Y_{1}(k a)-Y_{1}(k b)+G(k b)\right] k_{26}^{2}}{[P(k b)-Q(k b)+\ln a / b] k b Y_{2}(k b)+\left[Y_{1}(k a)-Y_{1}(k b)+G(k b)\right]\left[P(k b)-k b P^{\prime}(k b)+k_{26}^{2}\right]},
\end{gathered}
$$

which is the desired equation for the determination of $k$ and of $\omega$ via Eq. (17) $)_{1}$.

\section{NUMERICAL RESULTS}

For a rotated Y-cut quartz, ${ }^{9}$

$$
\begin{aligned}
& C_{66}=29.01 \mathrm{GPa}, \\
& e_{26}=-0.095 \mathrm{C} / \mathrm{m}^{2}, \\
& \epsilon_{22}=39.82 \times 10^{-12} \mathrm{C} / \mathrm{V} \mathrm{m},
\end{aligned}
$$

frequency equations (35) and (51) are solved numerically, and the first four frequencies are depicted in Figs. 4 and 5, respectively. In Figs. 4 and $5, R=(a+b) / 2$ is the average radius of the cylinder. Keeping $2 h=b-a$ fixed and letting $a \rightarrow \infty$, we see that in each case the resonant frequencies of the cylindrical shell approach those of the flat plate given by (22).

\section{CONCLUSIONS}

We have studied analytically the axial and the tangential thickness shear vibrations of a circular cylindrical piezoelectric shell, and have computed the first four resonant frequencies for a rotated Y-cut quartz shell.

\section{ACKNOWLEDGMENTS}

This work was supported by the U.S. Army Research Office Grant No. DAAH04-93-G-0214 to the University of Missouri-Rolla, and a matching grant from the Missouri Research and Training Center. The University of MissouriRolla awarded a subcontract to the Virginia Polytechnic Institute and State University.

${ }^{1}$ R. D. Mindlin, "Thickness-shear and flexural vibrations of crystal plates," J. Appl. Phys. 22, 316-323 (1951).

${ }^{2}$ H. F. Tiersten, "Thickness vibrations of piezoelectric plates," J. Acoust. Soc. Am. 35, 53-58 (1963).

${ }^{3}$ H. F. Tiersten, Linear Piezoelectric Plate Vibrations (Plenum, New York, 1969), pp. 86-93.

${ }^{4} \mathrm{~J}$. F. Haskins and J. L. Walsh, "Vibrations of ferroelectric cylindrical shells with transverse isotropy, I, radially polarized case," J. Acoust. Soc. Am. 29, 729-734 (1957).

${ }^{5}$ G. E. Martin, "Vibrations of longitudinally polarized ferroelectric cylindrical tubes," J. Acoust. Soc. Am. 35, 510-520 (1963).

${ }^{6} \mathrm{H}$. S. Paul, "Vibrations of circular cylindrical shells of piezoelectric silver iodide crystals," J. Acoust. Soc. Am. 40, 1077-1080 (1966).

${ }^{7}$ N. A. Shud'ga, A. Ya Grigorenko, and I. A. Loza, "Axisymmetric electroelastic waves in a hollow piezoelectric ceramic cylinder," Prikl. Mekh. 20, 26-32 (1984).

${ }^{8}$ A. E. H. Love, A Treatise on the Mathematical Theory of Elasticity (Dover, New York, 1944), 4th ed.

${ }^{9}$ H. F. Tiersten, Linear Piezoelectric Plate Vibrations (Plenum, New York, 1969), pp. 59-61. 